\title{
Analyse didactique clinique de l'activité décisionnelle de deux enseignantes en éducation physique et sportive (EPS)
}

L'éclairage du concept de milieu

Clinical didactic analysis of the decision-making activity of two teachers in

Physical and sports education: the lighting of the concept of "milieu"

\section{Marie-France Carnus}

\section{(2) OpenEdition}

\section{Journals}

Édition électronique

URL : http://journals.openedition.org/educationdidactique/554

DOI : 10.4000/educationdidactique.554

ISBN : 978-2-7535-1622-9

ISSN : $2111-4838$

\section{Éditeur}

Presses universitaires de Rennes

\section{Édition imprimée}

Date de publication : 1 octobre 2009

Pagination : 49-62

ISBN : 978-2-7535-1060-9

ISSN : 1956-3485

\section{Référence électronique}

Marie-France Carnus, « Analyse didactique clinique de l'activité décisionnelle de deux enseignantes en éducation physique et sportive (EPS) », Éducation et didactique [En ligne], 3-3 | Octobre 2009, mis en ligne le 01 octobre 2011, consulté le 08 décembre 2020. URL : http://journals.openedition.org/ educationdidactique/554; DOI : https://doi.org/10.4000/educationdidactique.554 


\title{
ANALYSE DIDACTIQUE CLINIQUE DE LACTIVITÉ DÉCISIONNELLE DE DEUX ENSEIGNANTES EN ÉDUCATION PHYSIQUE ET SPORTIVE (EPS) : L'ÉCLAIRAGE DU CONCEPT DE MILIEU
}

\author{
Marie-France Carnus, GRIDIFE, IUFM Midi-Pyrénées / \\ DiDiST - CREFI-T, Université Toulouse 3
}

\begin{abstract}
Résumé : Cet article a pour objet de présenter une étude transpositive centrée sur l'analyse didactique clinique de pratiques d'enseignants d'EPS en cours de gymnastique. Nous exposons dans un premier temps les options conceptuelles et méthodologiques, et plus particulièrement l'importation du concept évolutif de «milieu » initialement introduit par Brousseau dans la théorie des situations, et précisé plus tardivement comme «le système antagoniste du système enseigné » (1986). Cette importation entraîne un certain nombre d'adaptations qui se sont avérées nécessaires au regard de la particularité disciplinaire d'une part et, d'autre part, de la spécificité des questions de recherche centrées sur l'activité décisionnelle du sujet - enseignant. Dans un second temps, nous mettons en discussion de manière empirique les considérations précédentes au moyen d'extraits de corpus issus de deux études de cas : l'une en collaboration avec une enseignante débutante et l'autre avec une enseignante expérimentée. Nous montrerons en quoi le concept de milieu « revisité » permet de rendre compte des écarts entre intention et décision chez ces enseignantes ainsi que des effets de ces écarts sur les contenus enseignés.
\end{abstract}

Mots clés : didactique clinique de l'EPS, milieu didactique, Education Physique et Sportive, intention, décision.

\section{Introduction}

Les travaux que notre équipe mène au sein du DiDiST (Université Toulouse 3) nous ont progressivement conduits à construire un cadre théorique qui tente d'articuler les dimensions didactique et clinique pour l'analyse des pratiques d'enseignement-apprentissage en EPS. Cette orientation entraîne de fait une double vigilance épistémologique : celle d'une part liée à l'importation de concepts et notions issus d'autres didactiques et, d'autre part, celle de revisiter ces concepts et notions à la lumière des présupposés inhérents à la recherche clinique d'orientation psychanalytique. Dans cet article, nous mettons en discussion la pertinence du recours au concept de milieu pour l'analyse didactique clinique de l'activité décisionnelle de deux enseignantes d'EPS : l'une expérimentée et l'autre débutant dans le métier. À partir de résultats de recherches antérieures portant sur le processus décisionnel de l'enseignant d'EPS, nous focalisons l'analyse sur la question des écarts entre les intentions relatives aux contenus à enseigner et les décisions prises dans l'interaction d'où émergent les contenus effectivement enseignés. En prenant en compte la singularité des sujets observés et les particularités des contextes d'enseignement en EPS, nous éprouvons le caractère générique du concept de milieu afin de décrire et comprendre, chez les deux enseignantes, des régularités et des différences dans le passage des contenus à enseigner aux contenus réellement enseignés.

Présentation des travaux, problématique, emprunts conceptuels et méthodologiques

\section{Une recherche en didactique clinique de l'EPS}

L'approche ternaire ${ }^{1}$ caractéristique du didactique a pour particularité de questionner la spécificité des contenus disciplinaires au regard des conditions de leur transmission-appropriation. En Education Physique et Sportive (EPS), discipline scolaire, les contenus sont transposés des pratiques sociales de référence - les Activités Physiques Sportives et Artistiques (APSA) - ayant chacune leur propre logique, leurs évolutions et leurs enjeux de formation. Par ailleurs, l'environnement didactique de l'EPS comporte des particularités (lieux et 
espaces mouvants et spacieux, découpage temporel spécifique et contraignant, pratiques extra-scolaires des élèves, etc.) qui pèsent sur le fonctionnement didactique (Amade-Escot, 2005). Il en résulte une inéluctable distance entre les pratiques sociales et les pratiques scolaires qui interroge le processus de transposition didactique à différents niveaux. Du savoir à enseigner, au savoir effectivement enseigné jusqu'au savoir appris, les écarts constatés, en termes de remaniements, témoignent d'une complexité que seules les contraintes externes au système enseignant et/ou enseigné ne peuvent expliquer ou permettre de comprendre.

C'est cette complexité que nous cherchons à appréhender à différents niveaux de la « chaîne transpositive ». Les recherches menées par l'Équipe de recherche en Didactique Clinique de l'E.P.S. (EDiC) mettent en avant les limites des cadres et théories didactiques classiques pour rendre compte de certains phénomènes relatifs à la transmissionappropriation de savoirs. Aux influences extérieures agissant sur le système didactique, s'ajoutent des facteurs internes, inhérents aux logiques épistémiques propres des sujets engagés dans une relation asymétrique qui n'a de sens et de légitimité que par la médiation des savoirs qu'elle autorise et parfois qu'elle entrave. Nous entrons alors dans un domaine d'investigation plus intime prenant en compte la singularité des sujets dans leurs rapports aux savoirs. C'est ce parti pris, cette volonté de redonner aux sujets enseignants et apprenants une place centrale dans la relation didactique qui caractérise la dimension clinique des travaux de notre équipe (Terrisse, Carnus, 2009).

Cette dimension a pour conséquence directe de « sortir de l'ombre » l'activité des protagonistes du système didactique, l'enseignant et les élèves dans leur singularité, en tant que sujets « entièrement engagés » dans l'acte d'enseignement-apprentissage, avec ce qu'ils sont et ce qu'ils savent. L'approche clinique d'orientation psychanalytique en didactique se caractérise par un intérêt pour « le sujet singulier dans sa dynamique à la fois psychique et sociale » (Beillerot, Blanchard-Laville, Mosconi, 1996), des études « au cas par cas, au un par un » (Terrisse, 2000), ainsi que la prise en compte a priori du point de vue du sujet (Carnus, 2004). Ce positionnement, en rupture avec l'idée d'un sujet générique, épistémique, est alors de nature à questionner et orienter les choix conceptuels et méthodologiques des recherches engagées.

Postuler pour la singularité du sujet enseignant et le mettre au coeur des analyses didactiques amène le chercheur à se questionner autour d'une théorie du sujet en didactique : un sujet singulier du fait de son histoire personnelle, un sujet assujetti dans et par les institutions traversées, un sujet également divisé notamment entre ce qu'il dit qu'il fait et ce qu'il fait, ce qu'il faut qu'il fasse et ce qu'il ne peut s'empêcher de faire (Carnus, 2004). Ainsi, ce troisième présupposé intègre la dimension de l'inconscient, source de nombreuses divisions chez le sujet enseignant. Cette « hypothèse freudienne » (Sauret, 2000) permet, entre autre, d'éclairer la part d'insu dont nous avons fait à maintes reprises le constat - notamment dans le remaniement des contenus d'enseignement (Carnus, 2003).

Ainsi, à l'interface de la didactique et de la clinique - telle que nous l'avons définie - tout enseignement-apprentissage peut-être repensé dans le cadre d'un "modèle didactique systémique » (Astolfi, Develay, 1989) articulant trois logiques distinctes mais interdépendantes : celle de la discipline à enseigner qui ne peut échapper à une transposition permettant à l'élève l'accès à un savoir socialisé; celle du sujet apprenant construisant ses savoirs activement; celle enfin du sujet enseignant permettant et/ou facilitant cette construction en prenant un certain nombre de décisions au regard d'un « réseau d'intentions » complexes parmi lesquelles « l'intentio » qui renvoie aux orientations définies dans les programmes scolaires (Portugais, 1999). Nos travaux se focalisent sur l'activité décisionnelle de l'enseignant et plus particulièrement sur l'identification, la mesure et la conscience chez les enseignants des écarts entre les intentions relatives aux savoirs à enseigner et les décisions d'où émergent les savoirs « réellement » enseignés.

\section{Emprunts conceptuels et méthodologiques}

Figurent parmi les principaux concepts organisateurs de ces travaux et outils d'analyse des pratiques enseignantes, le plus souvent empruntés à la didactique des mathématiques, transposés et revisités 
dans le champ de la didactique clinique de l'EPS : la transposition didactique et le milieu.

\section{La transposition didactique questionnée}

Nos travaux interrogent la transposition didactique «interne "(Chevallard, 1985), c'est-à-dire « la part du processus de transposition qui advient dans le système didactique grâce aux transactions entre le système enseignant, le système étudiant et le système d'objets à enseigner et à étudier/apprendre » (Mercier, 2002). Nous nous centrons plus particulièrement sur « le passage d'un contenu de savoir précis à une version didactique de cet objet de savoir " (Verret, 1975; Chevallard, 1985). Cette phase de la transposition est « tout particulièrement l'affaire de l'enseignant » (Bru, 1991). En effet, « un contenu de savoir ayant été désigné comme savoir à enseigner subit dès lors un ensemble de transformations adaptatives qui vont le rendre apte à prendre place parmi les objets d'enseignement » (Chevallard, 1985).

Importée dans le champ de la didactique clinique de l'EPS (figure 1), cette partie du processus de transposition est revisitée d'une part par la prise en compte de la spécificité disciplinaire et, d'autre part, par l'intégration de la dimension du sujet. Le savoir à enseigner qui renvoie à l'intention didactique relève de plusieurs influences (Martinand, 2001) ${ }^{2}$ qui sont autant de références (Terrisse, 2001) pour le sujet enseignant. Nous identifions au moins trois sources différentes dans ces influences (Carnus, 2004): interne, liée à l'expérience et à l'expertise de l'enseignant; institutionnelle relative aux programmes et à leur interprétation par l'enseignant ${ }^{3}$; sociale au regard de la pratique sociale de référence, sa représentativité, son caractère plus ou moins scolarisable, son utilité perçue, son évolution, etc. C'est donc au carrefour de ces influences que s'élabore l'intention didactique relative au savoir à enseigner (1). On comprend mieux alors d'une part, les variabilités intra et inter individuelle dans les intentions didactiques qui orientent ensuite les décisions interactives d'où émerge le savoir enseigné (2). Toutefois, le savoir appris relève de la décision de l'apprenant (3). Cette tentative de schématisation permet nous semble-t-il de dépasser une vision linéaire de la transposition didactique et met au centre de la problématique la question des écarts. Les travaux que nous présentons ici se focalisent sur la mesure et la conscience chez les enseignants des écarts entre savoir à enseigner et savoir enseigné.

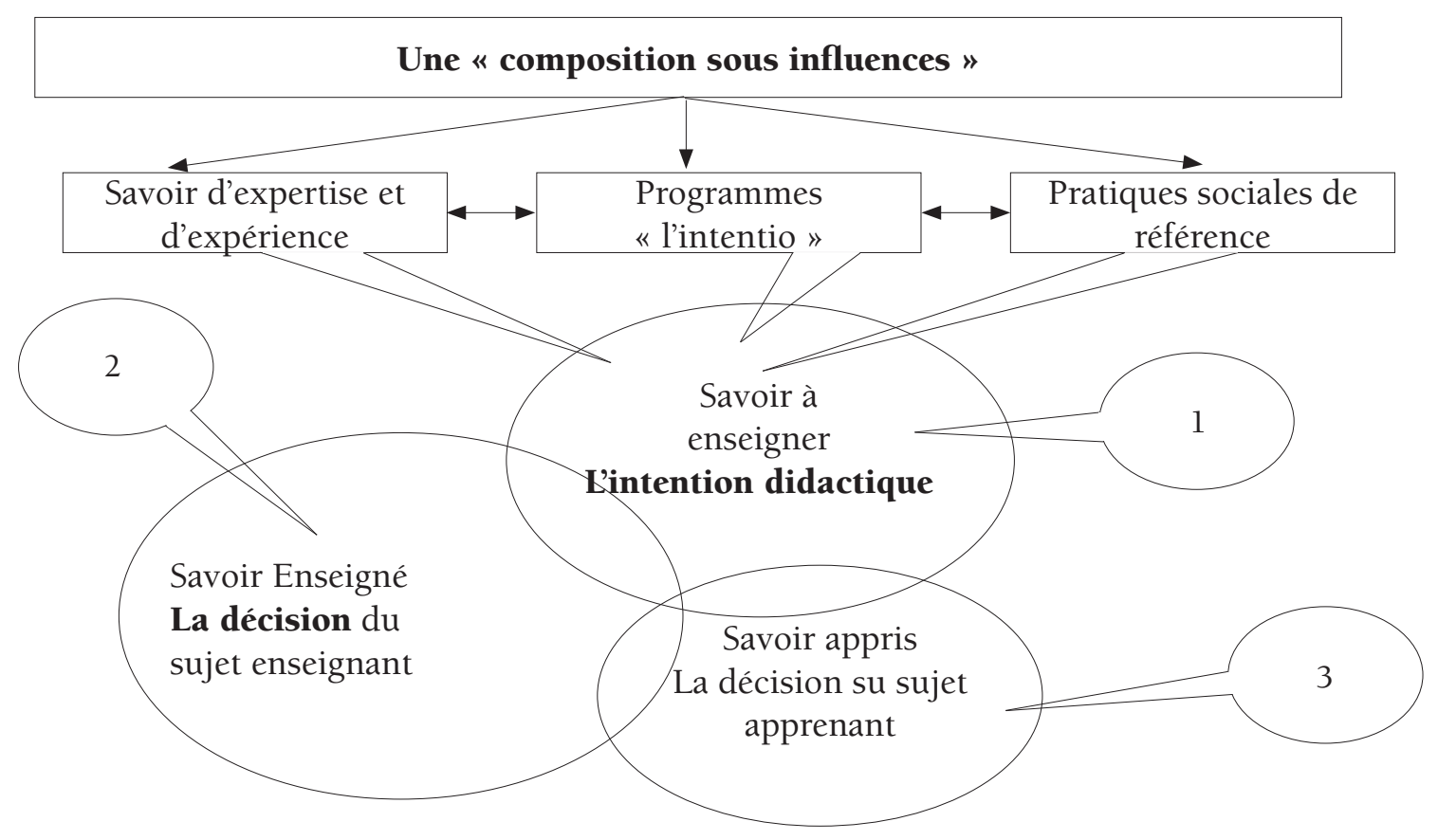

Figure 1 : schématisation de la transposition didactique en didactique clinique de l'EPS. 
Le milieu : un outil d'analyse des écarts

En tant que "système antagoniste du système enseigné " (Brousseau, 1986) puis «système des objets qui déterminent les pratiques de l'étude des savoirs " (Brousseau, 1996) le milieu peut être considéré comme " tout ce qui agit sur l'élève et ce sur quoi l'élève agit » (Brousseau cité par Perrin-Glorian, 1994). Il apparaît comme un concept nécessaire pour «prendre en compte le fonctionnement didactique » (Brousseau, 1986). Comme l'a souligné Perrin-Glorian (1999), « beaucoup de chercheurs se sont intéressés à ce concept évolutif » (de manière non exhaustive : Artaud, 1998; Brousseau, 1986, 1988; Chevallard, 1992, 1999; Brousseau, Centeno, 1991; Mercier, 1998; Perrin-Glorian, 1999; etc.). Importé de la théorie piagétienne dans la théorie des situations, le milieu est utilisé à deux échelles : une échelle globale, rattachée à l'idée de situation fondamentale où il renvoie à l'apprentissage d'un savoir et une échelle locale permettant l'étude d'une situation au moyen de la structuration du milieu (Perrin-Glorian, 1999; Margolinas, 1995, 1999). Dans la théorie anthropologique, le milieu renvoie à « l'ensemble des objets transparents pour les acteurs de l'institution » (Perrin-Glorrian, 1999). Dans cette optique, le milieu n'est pas défini en référence à une situation ou à un apprentissage. Il l'est « en référence à une institution et à une temporalité institutionnelle » (Chevallard, 1992). Comme le souligne Perrin-Glorian (1999), ces différentes visions du concept ne sont pas incompatibles et peuvent s'articuler. Elles permettent, selon nous, d'en entrevoir plusieurs aspects :

- des aspects structurels renvoyant à l'ensemble des objets matériels et symboliques - notamment langagiers - qu'il renferme;

- des aspects fonctionnels au travers des enjeux de savoir que sa conception et sa médiation permettent d'enseigner et d'apprendre;

- des aspects dynamiques qui émanent de la succession temporelle de ses différents états. Avant l'interaction, au moment de sa conception par l'enseignant, l'analyse a priori permet d'extraire les enjeux de savoirs à enseigner. Pendant l'interaction et durant toute la durée de la situation d'enseignement-apprentissage, à l'épreuve des faits didactiques, l'analyse a posteriori rend compte des savoirs réellement enseignés.
Entendu comme un système de médiations se transformant dynamiquement, le terme de mésogenèse (Chevallard, 1992) adopté par un certain nombre de didacticiens comparatistes (Mercier, Schubauer-Léoni, Sensevy, 2002) nous semble être un outil heuristique pertinent pour rendre compte des évolutions du milieu, de sa conception à sa mise en place et sa régulation par l'enseignant.

Ainsi, à différents moments de la transposition didactique, et plus particulièrement celui du passage des savoirs à enseigner aux savoirs réellement enseignés, nous cherchons à vérifier en quoi l'utilisation du concept de milieu en didactique clinique de l'EPS peut être un outil pertinent d'analyse des pratiques enseignantes et comment ce concept issu de la didactique des mathématiques pourrait permettre de rendre compte en EPS des écarts entre intentions et décisions et des effets de ces écarts sur les savoirs en jeu.

\section{Méthode et résultats}

Dans ces travaux, nous utilisons la méthodologie d'ingénierie didactique qui en tant que méthode de recherche n'a pas pour but ici de valider un enseignement ou d'en mesurer les effets (Artigue, 1990). Sa fonction essentielle est de créer un contexte d'observation favorable à l'étude et à la compréhension du fonctionnement didactique. Par ailleurs, son schéma chronologique en quatre phases (analyses préalables, conception et analyse a priori, mise à l'épreuve, analyse a posteriori et validation interne) permet de baliser et réorienter les étapes de la recherche. Pour le chercheur, ce choix méthodologique consiste à proposer puis à négocier un script didactique avec les enseignants associés à la recherche et d'en suivre les évolutions sur plusieurs séances. Bien que contraignante, cette option permet d'accéder de manière plus précise aux intentions des enseignants et rend moins aléatoires les rapprochements inter et intra site. La mise en tension entre l'analyse a priori et l'analyse a posteriori permet d'accéder aux écarts entre intentions et décisions. Plusieurs états successifs du milieu peuvent donc être analysés grâce à cette méthodologie.

Le premier état renvoie au milieu tel qu'il est conçu par le chercheur au temps 0 . La conception 
de l'ingénierie s'appuie sur la sélection de variables macro et micro didactiques jugées pertinentes au regard des savoirs en jeu ${ }^{4}$. Ces variables forment l'ossature d'un script didactique qui est ensuite négocié avec les enseignants associés. Cette négociation se déroule au cours de deux entretiens et consiste à reprendre une à une les variables sélectionnées et éventuellement les modifier au regard de l'enseignement usuel des enseignants collaborateurs. Une condition nécessaire au bon déroulement de la mise à l'épreuve est de veiller à la distance entre les options initiales de l'ingénierie et l'enseignement usuel des collaborateurs. Après négociation, au temps 1, l'analyse a priori du milieu permet d'accéder aux intentions des enseignants (deuxième état du milieu). Il ne s'agit pas de valider des contenus, le script est prétexte à l'analyse des pratiques enseignantes. Enfin dans un dernier temps, le milieu mis en place par l'enseignant et régulé pendant toute la durée de la situation d'enseignement - apprentissage est analysé (troisième état).

\section{Présentation du corpus}

Nous éclairons empiriquement les considérations qui précèdent par l'étude d'extraits de corpus issus de deux séances d'EPS conçues et mises en œuvre dans le cadre de cette méthodologie. Les savoirs en jeu sont identiques dans les deux séances et renvoient à l'enseignement-apprentissage d'un élément clé en gymnastique « l'appui tendu renversé » (figure 2) qui se caractérise par une double construction : celle des alignements bras - tronc - jambes à l'appui manuel renversé et celle de nouveaux repères visuels et proprioceptifs dans cette position inhabituelle.

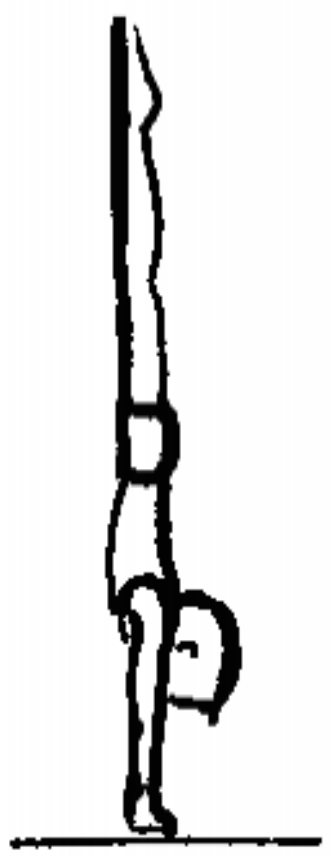

Figure 2 : l'appui tendu renversé

Ces contenus sont considérés par la plupart des enseignants d'EPS comme "un passage obligé », un incontournable qui figure d'ailleurs dans les programmes du cycle central du collège. Les deux séances se déroulent dans deux établissements différents avec des élèves de $5^{\text {e }}$ et deux ensei- gnantes volontaires que nous nommerons A et B. L'enseignante A est une enseignante expérimentée qui a 35 ans d'ancienneté. Elle est de surcroît spécialiste dans l'activité gymnastique. L'enseignante B est une enseignante débutante, professeur stagiaire également spécialiste dans l'activité. Bien que diffé- 
rents, les contextes d'enseignement-apprentissage ont des caractéristiques communes: classes de cinquième, même effectif, niveau équivalent, bon climat de classe, conditions matérielles correctes. Les deux enseignantes ont des conceptions relativement proches de la gymnastique.

Les extraits de corpus focalisent l'activité des deux enseignantes à deux moments différents : avant l'interaction avec les élèves, ce qui renvoie à l'intention relative aux savoirs à enseigner et pendant l'interaction permettant, au regard des décisions prises, d'accéder aux savoirs réellement enseignés. Au temps 0 et dans les deux cas, le dispositif initial qui contient les options de l'ingénierie est identique. Il est négocié par le chercheur avec les enseignantes au temps 1 avant d'être mis en œuvre avec les élèves au temps 2. Dans chaque site, nous étudions les différents « milieux» au travers de deux analyses : a priori aux temps 1 et a posteriori au temps 2 . Le rapprochement des deux analyses rend compte des écarts entre intention et décision et des effets de ces écarts sur les savoirs en termes de remaniements.
Par ailleurs, des entretiens « d'après-coup », réalisés avec les enseignantes quelques semaines après ont pour objectif d'accéder à leurs réseaux de signification. Ils visent également, au cas par cas, le repérage de la part d'insu dans le remaniement des contenus d'enseignement. L'approche clinique telle que nous l'avons caractérisée au début de cet article révèle dans ces travaux toute sa pertinence.

\section{Présentation de la situation initiale : les options de l'ingénierie (temps 0)}

Cette situation, classique en gymnastique, renvoie à un enseignement usuel. L'enjeu est, pour des élèves débutants ${ }^{6}$ ayant déjà construit la solidité des appuis mains, de passer d'appuis pédestres (départ debout, en fente avant, bras « collés aux oreilles » dans le prolongement du tronc) à des appuis manuels (bras tendus) en conservant l'angle bras - tronc ouvert.

Pour cela, le milieu initial qui sera négocié avec les enseignantes est le suivant (figure 3) :

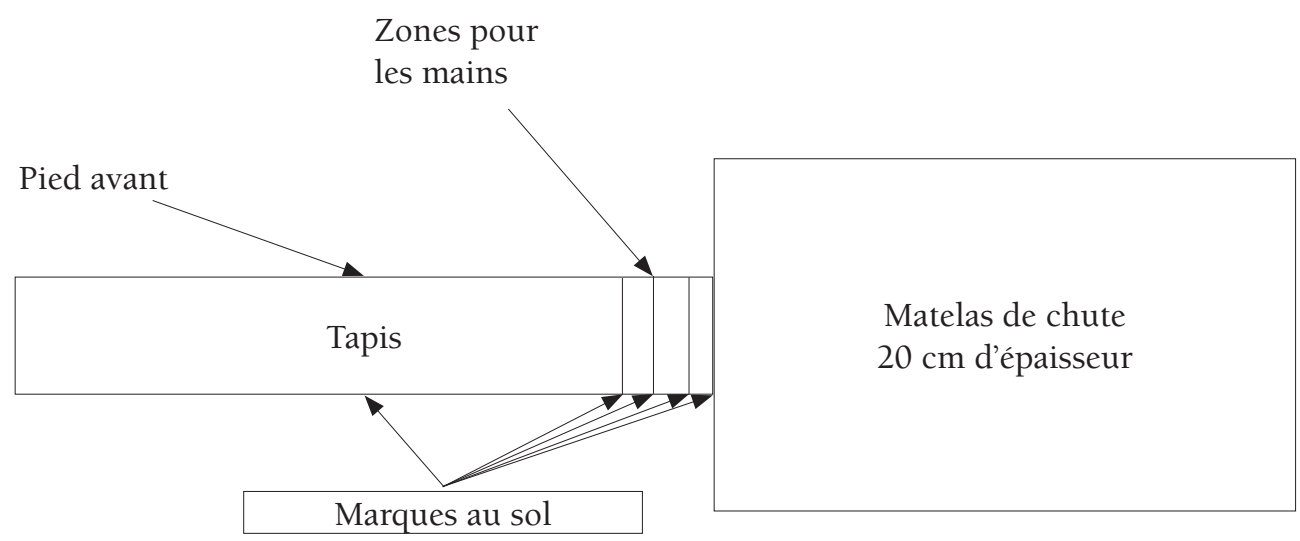

Figure 3 : le dispositif initial

Au-delà des objets matériels, d'autres éléments vont venir structurer le milieu, notamment les consignes relatives à « ce qu'il y a à faire » et la présence de parades sur les côtés à l'endroit de la pose des mains. Les consignes mentionnées dans le script didactique sont les suivantes : " de la fente avant, bras dans le prolongement du corps, poser les mains dans les secteurs les plus éloignées du pied avant situé derrière la première marque au sol. Appuyer très fort sur les mains, bras tendus pendant toute la durée du mouvement. Répéter l'exercice au moins 15 fois ${ }^{6}$.

Les aspects structurels du milieu ainsi conçu par le chercheur permettent de viser la construction par 
l'élève du maintien de l'alignement bras - tronc dans le passage de l'appui pédestre à l'appui actif manuel bras tendus (impulsion bras). Il permet aussi par son agencement une centration sur le dosage de la poussée de la jambe avant dans la bascule du corps. Enfin, il induit un repérage visuel sur la zone avant où vont se poser les mains.

\section{Temps 1 : négociation, analyse a priori et accès aux intentions des enseignantes}

Enseignante A (expérimentée) : les extraits qui suivent permettent d'identifier les modifications structurelles apportées au milieu par l'enseignante A au cours de l'entretien de négociation se déroulant deux jours avant la séance filmée.

"Si j'ai bien compris ici on ne s'occupe que de l'alignement des bras et ce qui est important c'est les zones..."

"Je mettrai un dima (gros matelas d'environ $60 \mathrm{~cm}$ d'épaisseur) derrière pour la sécurité... »

"Je sais que ça fausse un peu mais j'ai pas envie que certains se scratchent par terre..."

"J'pense pas que je leur dirai de partir les mains en haut... je trouve que c'est plus sécurisant en posant les mains par en bas...»

"J'aurai peut-être un peu de mal avec les parades, mais enfin, je vais essayer mais je sais pas trop comment. On verra au moment..."

Ces modifications structurelles du milieu apportées par l'enseignante A ne sont pas sans conséquence sur l'activité adaptative de l'élève que l'on peut analyser a priori. En effet, la médiation de ce « nouveau » milieu permet toujours la construction de l'alignement bras - tronc en position renversée mais cette construction se fait par l'ouverture (" par en bas ") de l'angle bras - tronc et non par le maintien de l'angle ouvert ("les mains en haut ») qui a priori est plus facile sur le plan technique mais plus difficile du point de vue de la prise de risque, autrement dit sur le registre affectif. De plus, la notion d'appui actif est absente du discours de l'enseignante. L'est-elle également dans l'intention? Par ailleurs, le remplacement d'un matelas de $20 \mathrm{~cm}$ d'épaisseur par un autre de $60 \mathrm{~cm}$ ( « le dima ») laisse présager que les élèves auront tendance à se laisser tomber sans trop de retenue sur ce matelas. Cet « excès » de sécurité pourrait avoir pour conséquence une absence de dosage de la poussée de la jambe avant dans la bascule du corps. Enfin, le repérage visuel, toujours focalisé sur la zone avant où les mains vont se poser, peut être empêché au moment de la pose des mains par une nécessaire flexion de la tête due à la présence du matelas. On peut toutefois constater que les effets de ces modifications structurelles du milieu sur les savoirs initialement en jeu sont - en partie - perçus par l'enseignante A ( "je sais que ça fausse un peu » ${ }^{7}$ ). Ces modifications sont prioritairement justifiées par des raisons de sécurité - leitmotiv récurrent chez cette enseignante du fait de son histoire singulière.

Enfin, la réserve émise sur les parades " je vais essayer mais je sais pas trop comment. On verra au moment..." laisse présager des adaptations de dernière minute et/ou dans l'urgence de l'interaction et par conséquent des écarts prévisibles entre intention et décision.

Enseignante B (débutante): les extraits qui suivent permettent de pointer les modifications structurelles apportées au milieu par l'enseignante B au cours de l'entretien de négociation se déroulant également deux jours avant la séance.

«Oui, je crois que j’ai bien compris. Il faut que les élèves restent alignés et bien insister sur la position en fente au départ, bras collés aux oreilles, je ferai des exos pendant l'échauffement pour qu'ils sentent bien la position de départ... »

"Je les mettrai plutôt face au mur avec le matelas contre le mur peut-être qu’ils auront plus confiance..."

«En fait ça change pas grand chose, je pense qu'ils auront moins peur et qu'ils s'engageront plus facilement... »

« 15 fois, en fait je me rends pas bien compte, ça me paraît beaucoup... Ils risquent d'en avoir vite marre... enfin on verra bien..."

"Je pense qu'il me faudra insister sur la parade mais en fait je ne sais pas trop quoi leur dire et la parade ça les branche pas trop... en fait dans la mesure où le matelas 
est au mur du coup on n'en a plus besoin de la parade, ça risque rien non?"

Là encore, les modifications structurelles du milieu initial apportées par l'enseignante B ont des effets sur les contenus que l'on peut analyser a priori. Concernant le maintien de l'alignement bras - tronc il reste au centre des intentions de l'enseignante B qui prévoit même un renforcement de la position de départ pendant l'échauffement. Toutefois, l'appui actif manuel n'est pas mentionné. Par ailleurs, le fait de mettre un matelas au mur est de nature à modifier l'activité adaptative des élèves qui pourraient avoir tendance à vouloir se renverser et se plaquer contre le matelas plutôt que de se concentrer sur le maintien postural bras dans le prolongement du corps. Ce nouvel aménagement peut également avoir pour conséquence un "surdosage » de la poussée de la jambe avant dans la mesure où l'appréhension de l'espace arrière est supprimée par la présence du matelas au mur qui, en cas de poussée trop importante, stoppe le mouvement. Là encore, le repérage visuel, initialement prévu en avant de la pose des mains risque d'être empêché par la présence du matelas contraignant la tête à une flexion (et par conséquent un regard vers l'arrière particulièrement déstabilisant du point de vue de l'équilibre). Contrairement à l'enseignante $\mathrm{A}$, ces modifications prévisibles touchant les savoirs en jeu ne semblent pas être perçues par l'enseignante B (« en fait ça change pas grand chose »). Le réaménagement du milieu est justifié par un engagement plus grand de la part des élèves du fait du risque perçu jugé moins important compte tenu de la présence du matelas au mur.

Enfin, les remarques portant sur le nombre de répétitions et sur les parades laissent planer une certaine incertitude autour de ces deux éléments structurant le milieu. Des décisions dans l'interaction sont prévisibles. S'inscriront-elles en continuité ou en rupture avec les intentions?

Temps 2 : observation de l'interaction, analyse a posteriori et accès aux décisions des enseignantes

Pour les deux sites, nous ne présentons ici qu'une sélection d'extraits significatifs au regard des modifi- cations structurelles apportées au milieu pendant la durée de la situation.

\section{Enseignante A :}

Après un échauffement d'une vingtaine de minutes pendant lequel l'enseignante $\mathrm{A}$ a rappelé aux élèves les notions d'alignement et d'ouverture bras - tronc, les élèves installent eux-mêmes le matériel suite aux explications de l'enseignante: " alors, le groupe 1, vous allez calmement chercher le gros dima, vous vous y mettez à tous les quatre sans faire les fous et vous l'amenez jusque-là... attendez, c'est pas fini, ensuite vous mettrez un tapis fin devant...».

Des consignes analogues sont données aux autres groupes pour l'installation du matériel. Lorsque le matériel du groupe 1 est installé, l'enseignante marque à la craie les différents repères pour la pose $\mathrm{du}$ pied et des mains. Puis elle regroupe les quatre élèves et leur donne la consigne suivante :

«Viens ici, Jonathan, tu vas montrer aux autres. Tu mets ton pied derrière cette marque, surtout, il faut pas la dépasser, et tu vas poser tes mains le plus loin possible au niveau des autres marques, allez, essaye, je te pare...»

Jonathan fait une première tentative, conformément à la consigne de l'enseignante. Il place son pied derrière la marque et va poser ses mains, par le bas, au niveau de la première marque. Son angle bras - tronc reste alors légèrement fermé. L'enseignante, à ce moment-là attrape une de ses jambes à la hauteur du genou, et l'amène à la verticale renversée en bloquant son dos pour finir d'ouvrir l'angle bras - tronc.

«Bon, voilà, il faut que tu sentes cette position, regardez les autres, oh, oh, regardez... » puis elle aide Jonathan à revenir les pieds au sol.

«C'est compris?»

Les élèves hochent de la tête.

«Alors vous faites ça une douzaine de fois, à tour de rôle... je vous laisse une craie pour repasser sur les marques quand elles s'effacent... allez c'est parti!!! » 
Les élèves se mettent au travail. La situation dure 14 minutes pendant lesquelles l'enseignante interviendra deux fois :

- une première fois à la huitième minute où après une brève observation de l'ensemble du groupe elle manipule chaque élève, l'un après l'autre en disant : "bon, tant pis si vous posez le pied un peu après la marque mais essayez de vous renverser sur le dima...»;

- une deuxième fois à la onzième minute, où elle manipule encore chaque élève en les encourageant " oui, voilà, c'est beaucoup mieux " pour le premier élève, "bon, c'est pas mal, mais pousse plus fort sur tes jambes " pour le deuxième, "bien, pas mal... " pour le troisième et enfin, " allez, allez, allez grandis-toi, encore, encore, là... ça vient, tu y es presque... " pour le quatrième.

Durant la situation, les différentes modifications du milieu ont des effets plus ou moins directs sur les contenus enseignés. Le dispositif matériel est certes conforme aux prévisions (matelas, tapis, marques à la craie). Toutefois, les médias utilisés (consigne verbale et démonstration par un élève) viennent structurer différemment le milieu. En effet, au début de la situation, lors de la présentation de la tâche à réaliser, l'accent est mis sur le positionnement du pied et des mains au regard des marques. Le fait de demander à l'élève d'aller le plus loin possible sans préciser la position initiale des bras laisse a priori aux élèves le choix des moyens pour y parvenir (soit "par le bas» par ouverture de l'angle bras - tronc, soit "par le haut " par maintien de l'ouverture bras - tronc.). Toutefois, la prestation de Jonathan devant les autres élèves hypothèque ce choix dans la mesure où la solution "par le bas " s'avère efficace dans la situation de démonstration-manipulation et qu'elle n'est ni invalidée ni commentée par l'enseignante. L'observation qui suit confirme cette analyse. Les autres élèves iront poser leurs mains "par le bas". Mais à ce moment-là, ils n'auront pas d'aide extérieure pour finir d'ouvrir l'angle bras - tronc. Ils ne pourront pas se renverser, leurs épaules resteront en avant des appuis mains et leur trajet se terminera la plupart du temps soit par une chute sur les genoux lorsque la poussée de la jambe avant sera insuffisante soit par une roulade sur le matelas en cas de surdosage de la poussée. La première intervention de l'enseignante à la huitième minute accentuera ce constat dans la mesure où l'avancée du pied a pour conséquence une plus grande fermeture de l'angle bras - tronc mais aussi une moindre appréhension des élèves qui se laisseront aller plus facilement dans l'espace arrière, diminuant les risques de chute sur les genoux. La deuxième intervention modifie profondément le milieu dans la mesure où les marques ne jouent plus leur rôle. La manipulation de l'enseignante est alors l'objet central du milieu, l'enjeu de savoir s'étant alors déplacé sur le passage des élèves à la verticale renversée.

\section{Enseignante B :}

Conformément à sa prévision, l'enseignante B a abordé, pendant l'échauffement la position de fente avant, bras placés en haut dans le prolongement du corps. À de nombreuses reprises elle a insisté sur l'alignement bras - tronc et sur le maintien de cet alignement dans le passage à l'appui manuel «alors, imaginez-vous que vous gardez cette position lorsque vous avez la tête en bas... ». Contrairement à l'enseignante A, l'enseignante $\mathrm{B}$ a installé elle-même le matériel avant le début de la leçon. Pour le groupe d'élèves concernés (groupe 1) par la situation, elle a placé un matelas contre un mur et un tapis devant. À la fin de l'échauffement, elle demande à chaque groupe de se diriger dans l'espace de leur atelier et donne au groupe 1 la consigne suivante :

«Bon alors tous les quatre, vous allez sur votre atelier et vous commencez à faire des ATR contre le mur en vous aidant... surtout vous faites bien attention... pensez à bien tendre vos bras... je vais venir vous voir dans un petit moment et je vous donnerai des consignes plus précises... allez, allez, ne perdez pas de temps».

Des consignes analogues sont données aux autres groupes. Elles ont pour objectif de répartir les élèves dans les différents ateliers et de les mettre en activité. Les élèves du groupe 1 , se dirigent vers leur atelier et après un temps de flottement de 2 à 3 minutes se mettent au travail. La situation dure 17 minutes pendant lesquelles l'enseignante intervient trois fois :

- une première fois à la sixième minute où elle trace les marques à la craie en disant « ici, vous mettez votre pied derrière, comme ça (l'enseignante se place en fente avant, les bras en haut et simule un début 
de bascule) et vous reprenez la position qu'on a vue à l'échauffement et vous allez poser les mains sur ces marques, là, le plus loin possible, vous risquez rien et surtout vous vous aidez... allez, vous le faites plusieurs fois, je reviens ";

- une deuxième fois à la onzième minute où elle se focalise sur le rôle de l'aide "regardez, pour vous aider, vous vous mettez sur le côté, un de chaque côté, mets-toi en face de moi (elle prend un élève par la main et le place en face d'elle). Cindy, allez vas-y, je t'aide, n'aie pas peur (dès que l'élève décolle les pieds du sol, elle lui attrape une jambe et vient le coller contre le matelas)... il faut surtout pas lui bloquer les pieds pour qu'elle puisse descendre, vous mettez vos mains, là... au bassin, c'est bon, tu peux revenir... alors une parade de chaque côté et à tour de rôle, je reviens... »;

- une troisième fois, à la quatorzième minute où elle manipulera deux élèves tout en supervisant le reste de la classe : " allez, à toi, vas-y, je t'aide (première manipulation), oh, dur, dur...»; " attention, Cindy, ne te met pas derrière tu vas te prendre un pied dans la tête, montre moi ce que tu fais... (manipulation) tu pousses pas avec tes jambes, il faut que j'aille te chercher, tends tes bras, tends tes bras... t'es pas assez forte sur tes bras...".

Là encore, les différentes évolutions structurelles du milieu ont des effets importants sur les contenus enseignés. Dès le début de la situation le dispositif matériel n'est pas conforme aux prévisions dégagées de la négociation. En effet, l'absence des marques au sol d'une part et la consigne donnée, d'autre part sont de nature à convoquer des enjeux de savoir différents de ceux qui étaient prévus. L'alignement bras - tronc disparaît pour laisser place à un renversement global contre le mur. Cet aménagement du milieu laisse aux élèves différents itinéraires pour réaliser la tâche (par le haut, par le bas, par le maintien de l'alignement, par l'ouverture de l'angle bras - tronc). Toutes ces possibilités n'ont pas le même degré de difficulté pour les élèves. De plus, au regard de leurs pré-acquis, certaines s'avèrent impossibles à mettre en œuvre (notamment l'ouverture de l'angle bras - tronc lorsque les appuis mains sont au sol). La première intervention de l'enseignante après 6 minutes de tâtonnement de la part des élèves oriente le choix des élèves dans le sens de l'intention initiale relative au maintien de l'ouverture bras - tronc (solu- tion par le haut). Dans les minutes qui suivent cette intervention, les élèves tentent la plupart de temps sans succès de répondre à la consigne. Leurs comportements sont alors analogues à ceux décrits dans le site $\mathrm{A}$. La deuxième intervention de l'enseignante ne porte pas sur les productions motrices des élèves - productions pourtant en inadéquation avec ses attentes. Elle déplace leur attention sur l'activité du pareur - sur laquelle elle avait quelques réserves au temps 1 . On peut aussi remarquer qu'à ce moment-là, les marques à la craie se sont effacées et qu'à nouveau les élèves vont tenter de se renverser contre le matelas de manière aléatoire. Enfin, la dernière intervention, tout comme sur le site $\mathrm{A}$, modifie à nouveau le milieu dans lequel la manipulation de l'enseignante devient l'objet central. À nouveau l'enjeu de savoir se déplace sur le renversement global.

\section{Conclusion : entre régularités et singularités}

Ces cas rapprochés au regard de l'étude des évolutions du milieu dans ses aspects structurels, fonctionnels et dynamiques mettent en évidence un certain nombre de régularités dans la description et la compréhension du fonctionnement didactique en EPS. En effet, dans les deux sites, nous rendons compte entre les temps 1 et 2 d'écarts entre intention et décision. Par ailleurs, ces écarts ont des effets plus ou moins conséquents sur les contenus d'enseignement qui sont en permanence remaniés par les modifications structurelles d'un milieu instable du fait de son " aménagement progressif... dont certaines dimensions ont été fixées aux premiers instants de la leçon »(Sensevy, Mercier, Schubauer-Léoni, 2000). Ainsi, le caractère labile du milieu rend contingents l'enseignement et l'apprentissage. Dans les deux sites également, cette analyse permet de mettre en évidence trois caractéristiques spécifiques du milieu au cours de l'interaction en EPS.

1/ Une centration des interactions didactiques des deux enseignantes davantage sur le résultat de l'action (le renversement) que sur les procédures et les processus (les actions à réaliser, les repères à construire). Cette régularité entre une enseignante débutante et une enseignante expérimentée interroge la profession d'autant que les deux enseignantes sont des spécialistes de l'activité ${ }^{8}$. On ne peut donc inférer ici un manque de maîtrise des contenus enseignés. Le 
mythe de "la magie de la tâche " (Marsenach, 1991) ou l'idée "qu'il suffit de mettre les élèves en situation pour qu'ils apprennent " seraient-ils à l'origine de ces de pratiques langagières massivement centrées sur le résultat de l'action et laissant une faible part aux interactions portant sur les critères de réalisation? On peut également faire l'hypothèse que l'expertise gymnique des deux sujets observés en lien avec un passé de gymnaste pourrait expliquer en partie cette régularité. Il serait intéressant de faire une étude similaire auprès d'enseignants non spécialistes dans l'activité.

2/ L'apparition au cours de l'interaction de nouveaux objets dans le milieu et notamment la manipulation par les enseignantes qui rend de ce fait obsolète le dispositif précédent. Ce constat est certainement à mettre en lien avec d'une part la spécificité des contenus disciplinaires en EPS essentiellement moteurs qui conduit les enseignants à des formes de pratiques ostensives mettant en jeu le corps selon diverses modalités (de la démonstration à la manipulation). Il est également à mettre en relation avec le caractère morphocinétique de l'activité gymnique dont les enjeux de formation s'articulent autour de la production de formes corporelles à la fois buts et moyens des apprentissages.

3/ La disparition ou l'effacement, en phase interactive, de certains éléments comme le nombre de répétitions ou encore la présence constante de l'aide et de la parade combinée avec les autres enjeux de savoirs. En effet, en ce qui concerne le nombre de répétitions, nous avons relevé dans le site $\mathrm{A}$, entre 4 et 8 répétitions par élève et, dans le site $B$, entre 3 et 6 . Nous sommes loin des 15 prévues dans le script et pas vraiment remises en question par les enseignantes. Cette variation interindividuelle entre les élèves souligne la contingence de l'apprentissage d'une habileté fermée qui nécessite un nombre conséquent de répétitions. D'autres séances seront alors nécessaires pour acquérir les savoirs nécessaires à la construction de la verticale renversée. D'autre part, dans les deux sites, la présence permanente de l'aide et de la parade combinée avec les autres enjeux de savoir est remise en question. Alors que l'enseignante expérimentée l'évacue du milieu sauf dans les moments interactifs avec les élèves où elle la prend totalement à sa charge, l'enseignante expérimentée tente de l'introduire, à moment donné pour la dévoluer aux élèves. L'aide et la parade deviennent alors les seuls enjeux de savoir sur lesquels elle se focalise. Ces constats soulignent la difficulté récurrente des enseignants d'EPS de poursuivre plusieurs objectifs en même temps et notamment l'acquisition d'habiletés motrices qui demandent du temps et un nombre de répétitions conséquent et la construction de savoir faire sociaux inscrits dans les programmes scolaires. 


\section{NOTES}

1. «La relation didactique est constituée de l'ensemble des échanges entre les élèves, le savoir et le professeur. Il s'agit d'une relation ternaire» (Jonnaert, P., Vander Borght, C. 1999, p. 178)

2. D'où l'expression « composition sous influence » (Martinand, 2001)

3. Portugais (1998) opère une distinction entre « l'intentio » (le projet social d'enseignement caractérisant les objets de savoir et leur sens), l'intentionnalité (l'intention privée de l'enseignant qui met en marche les positions intentionnelles) et les intentions didactiques qui sont mises en jeu dans une situation donnée pour déterminer un bon fonctionnement des problèmes à résoudre.

4. Cette sélection se fait à partir de travaux antérieurs ayant validé l'efficience des options retenues (Carnus, Terrisse, 2000). Les situations proposées dans le script sont des situations relativement « ordinaires » car usuellement mises en place par les enseignants.

5. Cette situation ne s'adresse qu'aux élèves n'ayant pas encore construit l'alignement bras - tronc. Elle s'inscrit dans le cadre d'un enseignement différencié caractérisé par la présence de différents ateliers correspondant à des besoins différents d'élèves mis en évidence au cours d'une évaluation diagnostique.

6. Le nombre de répétitions retenue a été estimé au regard de la durée prévue de la situation (entre 20 et 30 minutes), durée bien supérieure à ce qu'il est coutume d'observer dans les séances ordinaires d'EPS. Néanmoins, le script ne comportant qu'une seule situation d'apprentissage cette durée est réaliste. Elle a fait l'objet de discussion avec les enseignantes qui ont souhaité maintenir cette contrainte.

7. En modifiant le dispositif mis en place par l'ingénierie, l'enseignante a conscience de transformer la situation et par là même les enjeux de savoirs. Elle développera ce point dans la suite de l'entretien.

8. L'analyse comparée de pratiques d'enseignants débutants et expérimentés met en avant que l'expérience professionnelle et l'expertise dans un domaine de spécialité sont des facteurs structurant les compétences professionnelles des enseignants (Carnus, Garcia-Debanc, Terrisse, 2008 ; Carnus, Garcia-Debanc, 2008) 


\section{BIBLIOGRAPHIE}

Amade-Escot, C. (2005). Milieu, dévolution, contrat. Regard de l'éducation physique. In M.H. Salin, P. Clanché et B. Sarrazy (dir) Sur la théorie des situations didactiques, 91-98. Grenoble : La pensée sauvage.

Artaud, M. (1998). Introduction à l'approche écologique du didactique. L'écologie des organisations mathématiques et didactiques. In Actes de la IXième école d'été de la didactique des mathématiques, Houlgate 1997 (éds ARDM), 101-139.

Artigue, M. (1990). Ingénierie didactique. Recherches en didactique des Mathématiques, 9/3, 283-307.

Astolfi, J.P., Develay, M. (1989). La didactique des sciences. Paris : PUF.

Beillerot, J., Blanchard-Laville C., Mosconi, N. (1996). Pour une clinique du rapport au savoir. Paris: L'Harmattan.

Blanchard-Laville, C., Chaussecourte P., Hatchuel F., Pechberty B. (2005). Recherche clinique d'orientation psychanalytique dans le champ de l'éducation et de la formation. Revue française de pédagogie, $\mathrm{n}^{\circ} 151$, 111-162.

Brousseau, G. (1986). Fondements et méthodes de la didactique des mathématiques. Recherches en didactique des mathématiques, 9/3, 309-336.

Brousseau, G. (1988). Le contrat didactique : le milieu. Recherches en didactique des mathématiques, 9/3, 309-336.

Brousseau, G. (1996). L'enseignant dans la théorie des situations didactiques. In R. Noirfalise, M.J. PerrinGlorian, Actes de la huitième école d'été de didactique des mathématiques, IREM de Clermont-Ferrand, 3-46.

Brousseau, G., Centeno, J. (1991). Rôle de la mémoire didactique de l'enseignant. Recherches en Didactique des Mathématiques, 11/2-3, 167-210.

Bru, M. (1991). Les variations didactiques dans l'organisation des conditions d'apprentissage. Toulouse : EUS.

Carnus, M.F., Terrisse, A. (2000). La prise en compte par l'enseignant des stratégies de repérage des élèves dans un apprentissage gymnique : l'ATR : une étude de transposition didactique. Science et Motricité, 40, 32-41.

Carnus, M.F. (2003). Analyse didactique du processus décisionnel de l'enseignant d'EPS en gymnastique : une étude de cas croisés. In: C Amade-Escot (éd) Didactique de l'éducation physique, état des recherches, 195-224, Paris : Revue EPS.

Carnus, M.F. (2004). La prise en compte du sujet dans la recherche en didactique, vers une didactique clinique: intérêt, difficultés, limites. $5^{\mathrm{e}}$ congrès international de l'AECSE d'actualité de la recherche, Paris, 31 Août et $1,2,3$ et 4 septembre.

Carnus, M.F., Garcia-Debanc, C. (2008). De l'analyse didactique de pratiques langagières à la spécification de compétences professionnelles d'enseignants débutants. Français et Education Physique et Sportive. Les dossiers des Sciences de l'Education, 20, 39-54.

Carnus, M.F., Garcia-Debanc, C., Terrisse, A. (2008). Analyses de pratiques des enseignants débutants, approches didactiques. Grenoble : La pensée sauvage.

Chevallard, Y. (1985). La transposition didactique. Grenoble : La pensée sauvage.

Chevallard, Y. (1992). Concepts fondamentaux de la didactique : perspectives apportées par une approche anthropologique. Recherches en didactique des Mathématiques, 12/1, 73-112.

Chevallard, Y. (1999). Analyse de pratiques enseignantes et didactique des mathématiques : l'approche anthropologique. In R. Noirfalise (éd), Analyse de pratiques enseignantes et didactique des mathématiques, Actes de l'université d'été de la Rochelle, 91-120, Irem de Clermont-Ferrand.

Jonnaert, P. Vander Borght, C. (1999). Créer des conditions d'apprentissage : un cadre de référence socioconstructiviste pour une formation didactique des enseignants. Bruxelles : De Boeck-Université.

Margolinas, C. (1995). La structuration du milieu et ses apports dans l'analyse a posteriori des situations. In C. Margolinas, Les débats de didactique des mathématiques, Annales 1993-1994, Grenoble : La pensée sauvage. 
Margolinas, C. (1999). Le milieu et le contrat, concepts pour la construction et l'analyse des situations d'enseignement. In R. Noirfalise (éd), Analyse de pratiques enseignantes et didactique des mathématiques, Actes de l'université d'été de la Rochelle, 3-16, IREM de Clermont-Ferrand.

Marsenach, J. (1991). EPS : quel enseignement? Paris : INRP.

Martinand, J.L. (2001). Pratique de référence et problématique de la référence curriculaire. In A. Terrisse (Ed) Didactiques des disciplines : les références au savoir. 179-204. Bruxelles : De Boëck.

Mercier, A. (1998). La participation des élèves à l'enseignement. Recherches en didactique des mathématiques, 18/3, 279-310.

Mercier, A. (2002). L'observation du travail des élèves quels en sont les objets élémentaires et comment peut-on les produire? In P Venturini, C. Amade-Escot, A. Terrisse (éds), Etude des pratiques effectives : l'approche des didactiques, 147-173. Grenoble : La pensée sauvage.

Mercier, A., Schubauer-Leoni, M.L., Sensevy, G. (2002). Vers une didactique comparée. Revue Française de Pédagogie, 141, Paris, INRP.

Perrin Glorian, M.J. (1994). Théorie des situations didactiques : naissance, développement, perspectives. In M. Artigue et al, Vingt ans de didactique des mathématiques en France. Grenoble: La pensée sauvage.

Perrin Glorian, M.J. (1999). Problèmes d'articulation de cadres théoriques : l'exemple du concept de milieu. Recherches en didactique des mathématiques, 19/3, 279-322.

Portugais, J. (1999). Esquisse d'un modèle des intentions didactiques. Actes des secondes journées didactiques de La Fouly, 57-88.

Sauret, J.M. (2000). Freud et l'inconscient. Les éditions Milan.

Sensevy, G., Mercier, A., Schubauer-Leoni, M..L. (2000). Vers un modèle de l'action didactique du professeur. A propos de la course à 20, Recherches en didactique des mathématiques, 20/3, 263-304.

Terrisse, A. (2000). Epistémologie de la recherche clinique en sport de combat. In A. Terrisse (dir.) Recherches en sports de combat et en arts martiaux, 95-108. Paris : Revue EPS.

Terrisse, A. (2001) La référence dans l'enseignement de l'éducation physique et sportive. In A. Terrisse (Ed) Didactiques des disciplines : les références au savoir. 119-139. Bruxelles : De Boëck.

Terrisse, A., Carnus, M.F. (2009). Didactique clinique de l'EPS, quels enjeux de savoirs ? Bruxelles : De Boëck.

Verret, M. (1975). Le temps des études. Paris : Champion. 